INPLASY

PROTOCOL

To cite: Yeye et al. The development and use of sport coaches' reflection: a systematic review. Inplasy protocol 202130034. doi: 10.37766/inplasy2021.3.0034

Received: 11 March 2021

Published: 11 March 2021

Corresponding author:

Felipe Yeye

feyeye@gmail.com

Author Affiliation: University of Porto, Faculty of Sport

Support: No financial support.

Review Stage at time of this submission: Preliminary searches.

Conflicts of interest: None declared.

\section{The development and use of sport coaches' reflection: a systematic review}

\author{
Yeye, F1; Afonso, J2; Araújo, R³.
}

Review question / Objective: The purpose of this systematic review was to examine what is currently known about sport coaches' use and development of reflective practice.

Condition being studied: Sport coaches and their application of reflective skills.

Information sources: A comprehensive computerized search of the following eight electronic databases will be performed: Cochrane Library, EBSCO, Pub Med, Scielo, SCOPUS, and Web of Science. This search process had no restriction regarding year of publication. Boolean operators were applied into search the article title and abstract: ("reflect* OR reflex") AND ("coach* OR trainer") AND sport*. In addition, in the end authors will perform a manual search of studies' references included in the automatized search to find other articles not identified in the computerized search. The research team will then try to contact expert on these areas of research (coach education and reflective practice) so they can suggest other articles.

INPLASY registration number: This protocol was registered with the International Platform of Registered Systematic Review and Meta-Analysis Protocols (INPLASY) on 11 March 2021 and was last updated on 11 March 2021 (registration number INPLASY202130034).

\section{INTRODUCTION}

Review question / Objective: The purpose of this systematic review was to examine what is currently known about sport coaches' use and development of reflective practice.

Condition being studied: Sport coaches and their application of reflective skills. 


\section{METHODS}

Participant or population: Coaches of any sport with no restrictions regarding sex, age or context of intervention (professional, amateur, etc.).

Intervention: Interventions focused on the development or use of coaches' reflection practice.

Comparator: Comparators are not required.

Study designs to be included: No limitations imposed on study design.

Eligibility criteria: The present SR was conducted in accordance with the Preferred Reporting Items for Systematic Reviews and Meta-analysis (PRISMA; Moher et al. 2009). In particular, empirical articles were eligible for inclusion if published in in peer-reviewed international journals. In addition, Participants, interventions, comparators, outcomes, and study design (PICOS) were defined as followed: (i) coaches of any sport with no restrictions regarding sex, age or context of intervention (professional, amateur, etc.); (ii) interventions focused on the development or use of coaches' reflection practice; (iii) comparators not required (iv) assessments of the development of coaches' reflection skills; (v) no limitations imposed on study design. Studies were excluded if: (i) were not published in peerreviewed international journals; (iv) were not focused on reflective practice in sport's coach context (v) those who were not empirical studies, such as opinion articles, review articles.

Information sources: A comprehensive computerized search of the following eight electronic databases will be performed: Cochrane Library, EBSCO, Pub Med, Scielo, SCOPUS, and Web of Science. This search process had no restriction regarding year of publication. Boolean operators were applied into search the article title and abstract: ("reflect* OR reflex") AND ("coach* OR trainer") AND sport*. In addition, in the end authors will perform a manual search of studies' references included in the automatized search to find other articles not identified in the computerized search. The research team will then try to contact expert on these areas of research (coach education and reflective practice) so they can suggest other articles.

Main outcome(s): Analysis of the development of coaches' reflection skills.

Quality assessment / Risk of bias analysis: Methodologic quality of studies was assessed using the Mixed Methods Appraisal Tool MMAT; Hong et al., 2018).

Strategy of data synthesis: The initial searching of databases will be exported to reference manager software (EndNoteTM X9, Clarivate Analytics, Philadelphia, PA, USA). Duplicates will be then removed. The remaining articles will be then screened (title, abstract and full article if necessary) and removed if: not empirical articles, not focused on coaches' reflective practice, not in Portuguese, English or Spanish. In order to analyse all the information from the studies selected to this review, conventional content analysis will be used. To provide an analysis and to put into evidence the information that could give an answer to our aim, framework will be created a prior, which included: (i) purpose, (ii) participants and context, (iii) study design, (iii) data collection, (iv) data analysis, and (v) main results. Both authors will review the data synthesis and differences of opinion will be discussed and solved with the third author until consensus was achieved.

Subgroup analysis: In case of sufficient participants, some subgroup analysis can be done (for instance, compare expert and novices coaches, or professional and amateur levels).

Sensitivity analysis: Articles will not be excluded based on low scores. Instead, the scores will be used to weigh the confidence of each finding during the synthesis.

Country(ies) involved: Portugal and Brazil. 
Keywords: Reflection, reflective practice, reflective skills, coach education, coach.

Contributions of each author:

Author 1 - Felipe Yeye.

Author 2 - José Afonso.

Author 3 - Rui Araújo. 